\title{
Sulla realizzazione di due pendoli a breve periodo per sismografi orizzontali e verticali ad amplificazione ottico-galvanometrica
}

\author{
A. Girlainda
}

Ricevuto il 18 Aprile 1963

Riassunto. -. Vengono descritti due pendoli a breve periodo per sismografi orizzontali e verticali ad amplificazione ottico-galvanometrica del tipo Galitzin. In questa prima nota sono esposti i criteri seguiti per una facile costruzione dei pendoli e per la realizzazione delle unità sismometriche da tempo in funzione in alcune stazioni italiane.

Sumaky. - . There are described two short period pendulums for horizontal- and vertical- component seismographs (electromagnetic and optical magnification) of the Galitzin type. In this first paper there are explained the rules to follow for an easy construction of the pendulums and for the performance of the seismographical unities for a time already in function in some Italian Stations.

Nei sismografi elettromagnetici ogni unitì sismometrica, utilizzabile per la registrazione di una componente del moto del suolo, è costituita da tre elementi fondamentali: pendolo, galvanometro, registratore. Il sistema pendolare (a traslazione o a rotazione) genera una corrente d'induzione in un sistema di bobine che, o si sposta $\left({ }^{1}\right)$, solidalmente con la massa pendolare, nell'intraferro di un magnete (Galitzin), o, fissato al supporto $\left({ }^{2}\right)$, abbraccia un circuito magnetico la cui riluttanza varia per effetto delle oscillazioni pendolari (Benioff). I due tipi, pur costituendo due dispositivi strumentali diversi, conducono ad una identica trattazione teorica. Le equazioni a cui si perviene sono analoghe, e nel citarle, mi riferirò al primo dispositivo, anche perché i sismografi dei quali riferisco appartengono al primo tipo. La corrente prodotta dal movimento del pendolo viene, in entrambi i casi, inviata in un galvanometro a bobina mobile le cui deviazioni vengono registrate su un registratore fotografico. 
I rriteri di accoppiamento pendolo-galvanometro possono essere diversi, tenendo presente che il galvanometro reagisce sul pendolo. Questa circostanza potrebbe far pensare che, in ogni caso, sia indispensabile adottare criteri costruttivi e di circuito tali da elininare, o rendere trascurabile, la reazione galvanometrica, considerata come damnosa

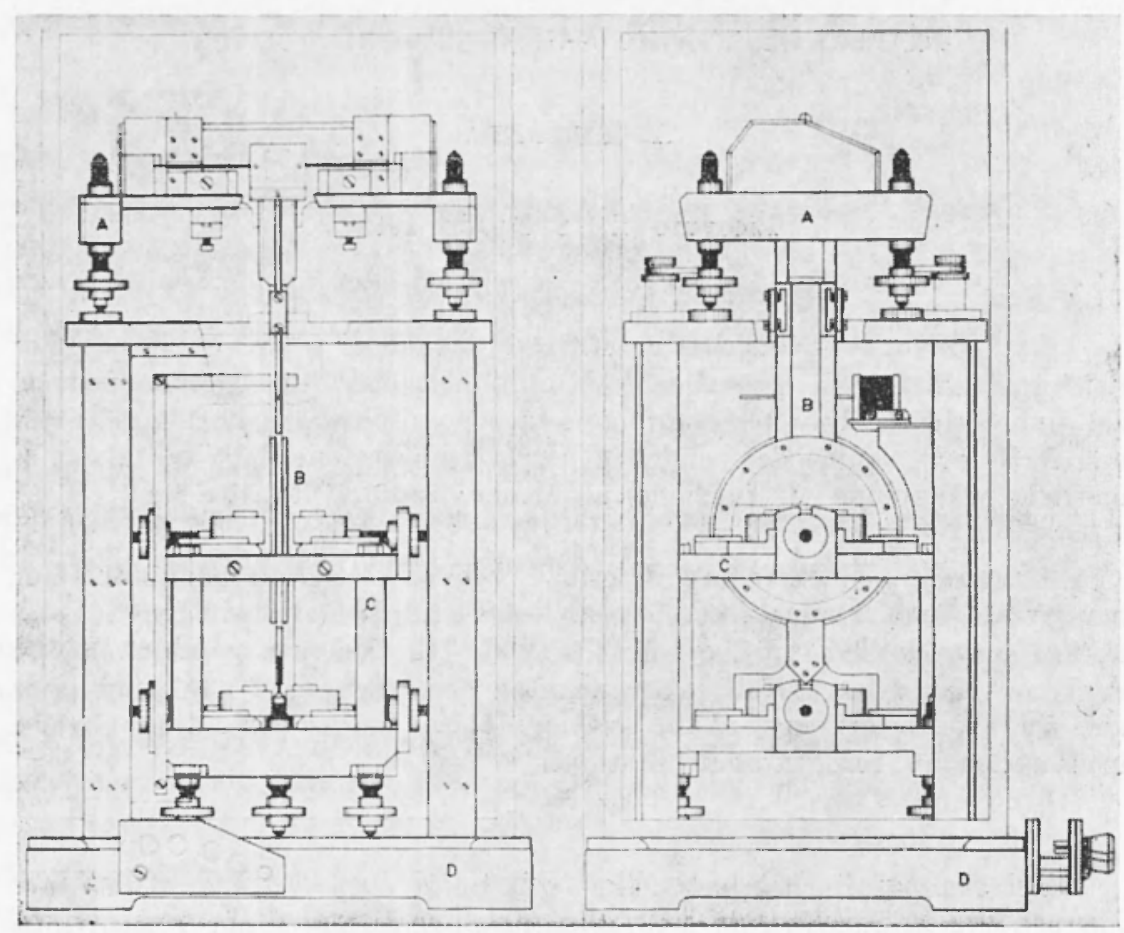

l'ig. ] - Sistena pendolare per la componente orizzontale visto di fronte e di fianco.

o, commenue, inproduttiva. Fu Wemner che per primo ha realizzato un sismografo sfruttando la reazione galvanometrica $\left(^{3}\right)$ che, secondo un'idea proposta successivamente da Grenet ("), potrebbe essere persino accentuata inserendo più galvanometri, alcuni dei quali impiegati soltanto cone generatori di reazioni.

I sismografi elettromagnetici a breve periodo, da tempo in funzione nella stazione sismica di Messina e in alcune stazioni impiantate in prossinità di grandi dighe nell'Italia del Nord, sono stati studiati e progettati in modo da semplificare notevolmente la costruzione dei sistemi pendolari. Tale costruzione non richiede mezzi speciali e può 
essere realizzata anche in una modesta officina, quale può essere quella di un Istituto universitario o Osservatorio.

I pendoli, aventi periodo proprio di $1 .^{\mathrm{s}}$, sono accoppiati con galvanometri aventi periodo proprio di 2 s. L'asse di rotazione del pendolo, facente parte dell'unità sismometrica utilizzata per la registrazione del-

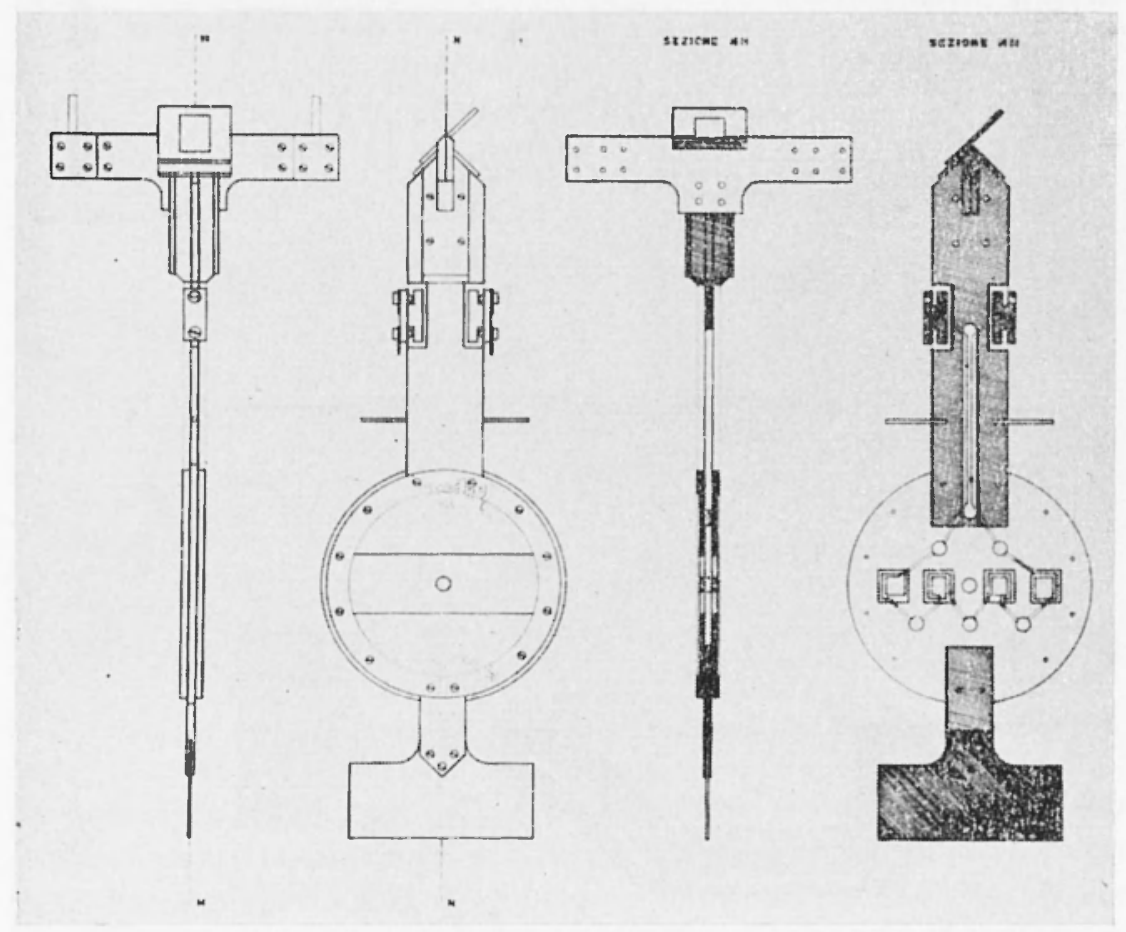

Fig. 2 - Braccio pendolare per la componente orizzontale.

la componente orizzontale, è realizzato mediante due laminette di acciaio aventi lo spessore di $0,05 \mathrm{~mm}$, con le quali il pendolo viene sospeso ad un robusto supporto superiore ( $A$, fig. 1) munito di tre viti di livello. Tale supporto è appoggriato su un sostegno costituito da un castello metallico con basamento di bronzo.

La massa pendolare è costituita da un braccio metallico portante un disco di materiale isolante nel quale sono incorporate quattro bobine per l'induzione elettromagnetica (fig. 2). Tali bobine sono facilmente asportabili e sostituibili con altre aventi la stessa massa, ma numero di spire diverso, in modo da poter realizzare, secondo gli scopi d'impiego, amplificazioni massime variabili in un intervallo sensibilmente più 
ampio di quello che comunemente si può ottenere influendo esclusivamente sulla distanza tra i magneti.

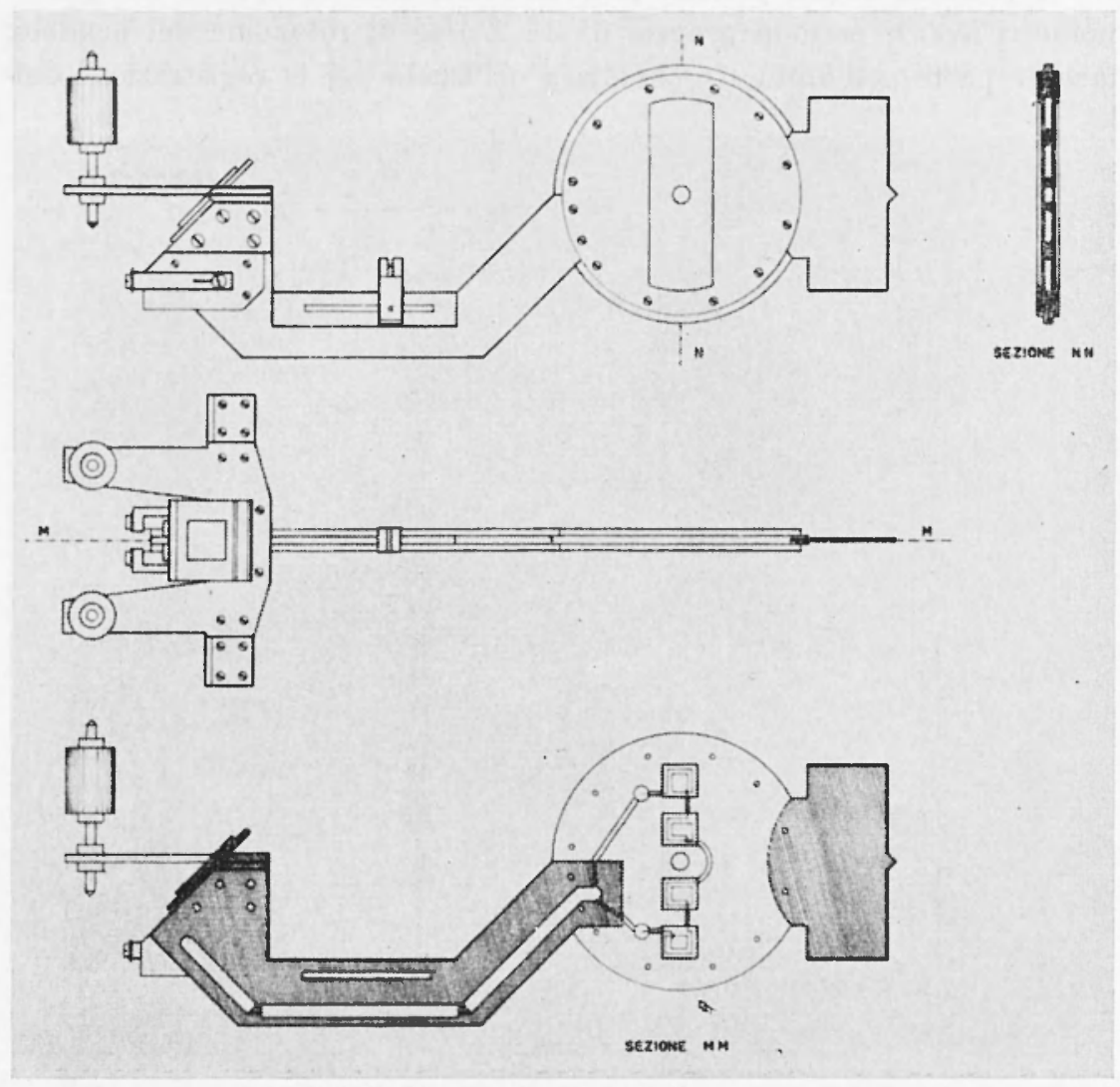

Fig. 3 - Braccio pendolare per la componente verticale.

Lo smorzamento è ottenuto mediante un'appendice di rame, solidale col disco, immersa nel campo magnetico generato da una coppia di magneti permanenti, sottostante la coppia di magneti utilizzata per il campo inducente. Le due coppie di magneti, fissate su slitte spostabili, sono sostenute da un pesante supporto (C, fig. 1) munito di viti di livello e appoggiato sul basamento. Ai margini di quest'ultimo è ricavata una scanalatura che consente l'alloggiamento di una custodia di perspex trasparente.

Nelle unità attualmente in funzione, il pendolo è accoppiato con un galvanometro avente, come si è detto, un periodo proprio (in assenza 
di smorzamento) di 2 s. Il galvanometro è del tipo a doppia bobina. Utilizzando una delle due bobine si ha la possibilità di ottenere sensibilità.

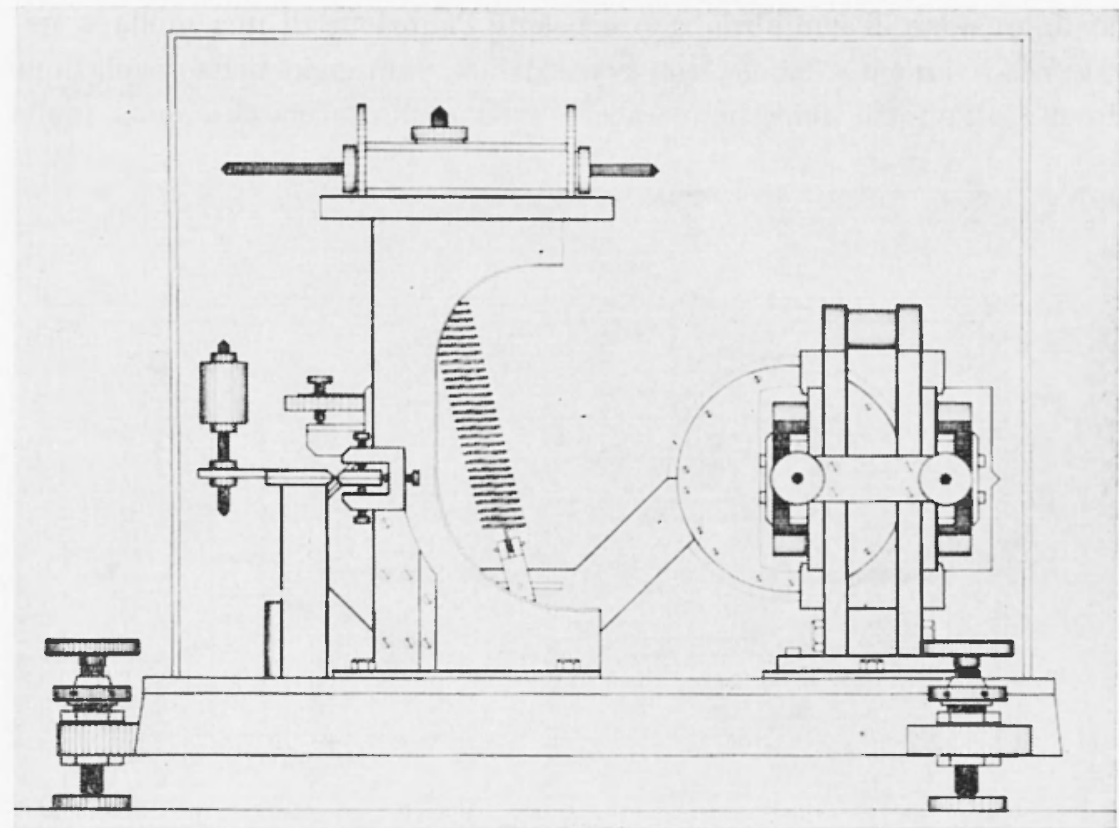

Fig. 4 - Pendolo per la componente verticale visto di fianco.

comprese tra 15 e $5 \cdot 10^{-9}$ e resistenze esterne, per il richiesto grado di smorzamento, fino a $200 \mathrm{ohm}$. L'altra bobina consente di ottenere sensibilità comprese tra 5 e $0,6 \cdot 10^{-9}$ per resistenze esterne fino a $20.000 \mathrm{ohm}$. Ho ritenuto vantaggioso l'uso di questo tipo di galvanometro in quanto è permessa, mediante opportuni shunt magnetici di cui il galvanometro è dotato, la regolazione della resistenza esterna necessaria per raggiungere il regime di smorzamento voluto, tenuto conto dei diversi sistemi di bobine pendolari adottabili. Evidentemente l'adozione di tale tipo di galvanometro non è indispensabile; altri tipi possono essere utilizzati, secondo le disponibilità, purché il periodo proprio dello strumento sia compreso in un intervallo la cui ampiezza dipende dal periodo proprio del pendolo, volendosi limitare alla realizzazione di un sismografo equivalente ad un sistema Galitzin.

Nell'unità sismometrica utilizzata per la registrazione della componente verticale, la parte pendolare è realizzata mediante un braccio di ottone portante, nella sua estremiti, un disco con le bobine incorporate 
analogo a quello già descritto (fig. 3). La massa pendolare è distribuita in modo da realizzare un notevole momento d'inerzia rispetto all'asse di rotazione, pur richiedendo un debole momento di richiamo verso la configurazione di equilibrio. Ció consente l'adozione di una molla a spirale relativamente debole, con conseguente vantaggio nella regolazione dovuta all'effetto della temperatura sulla costante elastica della molla

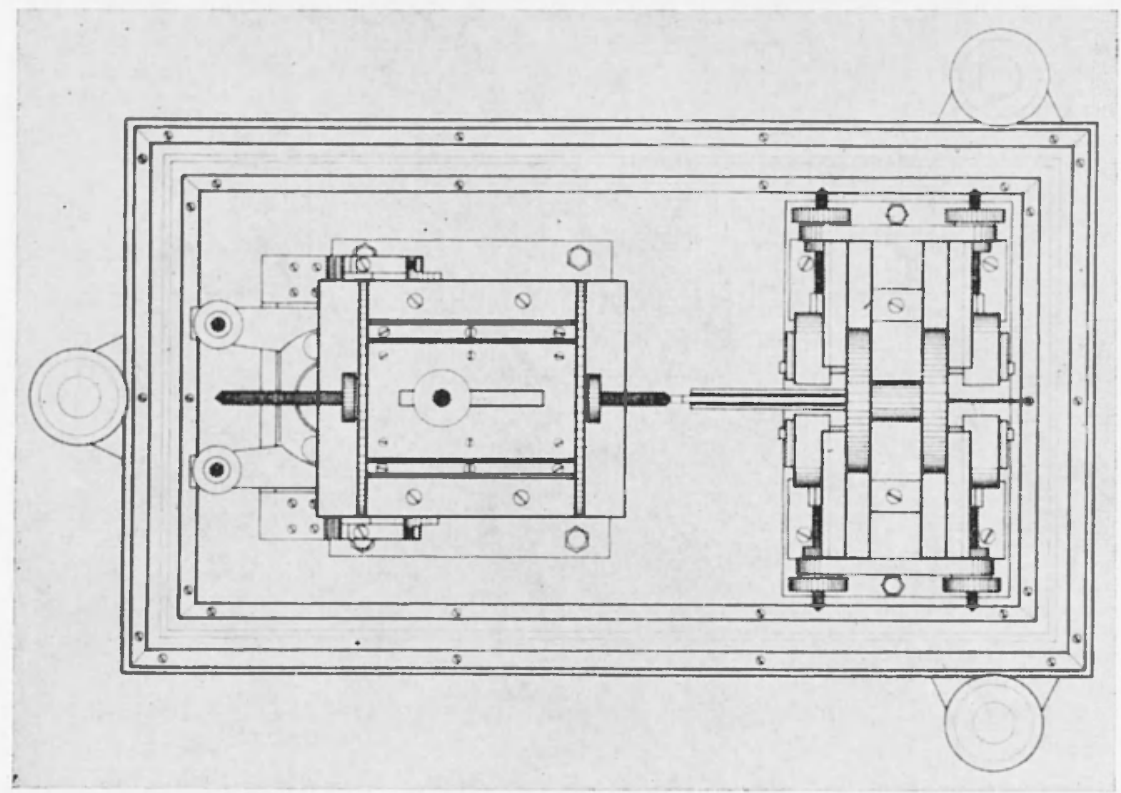

Fig. 5 - Pendolo per la componente verticale visto di sopra.

stessa. Il braccio porta, nella sua estremità posteriore, due masse addizionali di ottone, spostabili verticalmente. L'attacco della molla al braccio è spostabile lungo un'apposita scanalatura, in modo da poter variare facilmente, secondo le necessiti, il momento della forza elastica generata dalla molla stessa.

Una piattaforma di bronzo (cm $60 \times 35)$, munita di tre viti di livello e di una scanalatura ai margini per il collocamento della custodia di perspex trasparente (figg. 4, 5, 6), sopporta un primo ponte per l'attacco del braceio pendolare, un secondo ponte per l'attaceo al supporto della molla a spirale ed un complesso di slitte con due coppie di magneti permanenti spostabili mediante viti laterali. L'asse di rotazione è determinato dalle solite due laminette di acciaio che costitniscono il cardine del braccio pendolare. Quest'ultimo può essere agevol- 
mente bloccato mediante due ganasce laterali, munite di viti di blocco, solidali col ponte che serve per l'attacco della molla a spirale.

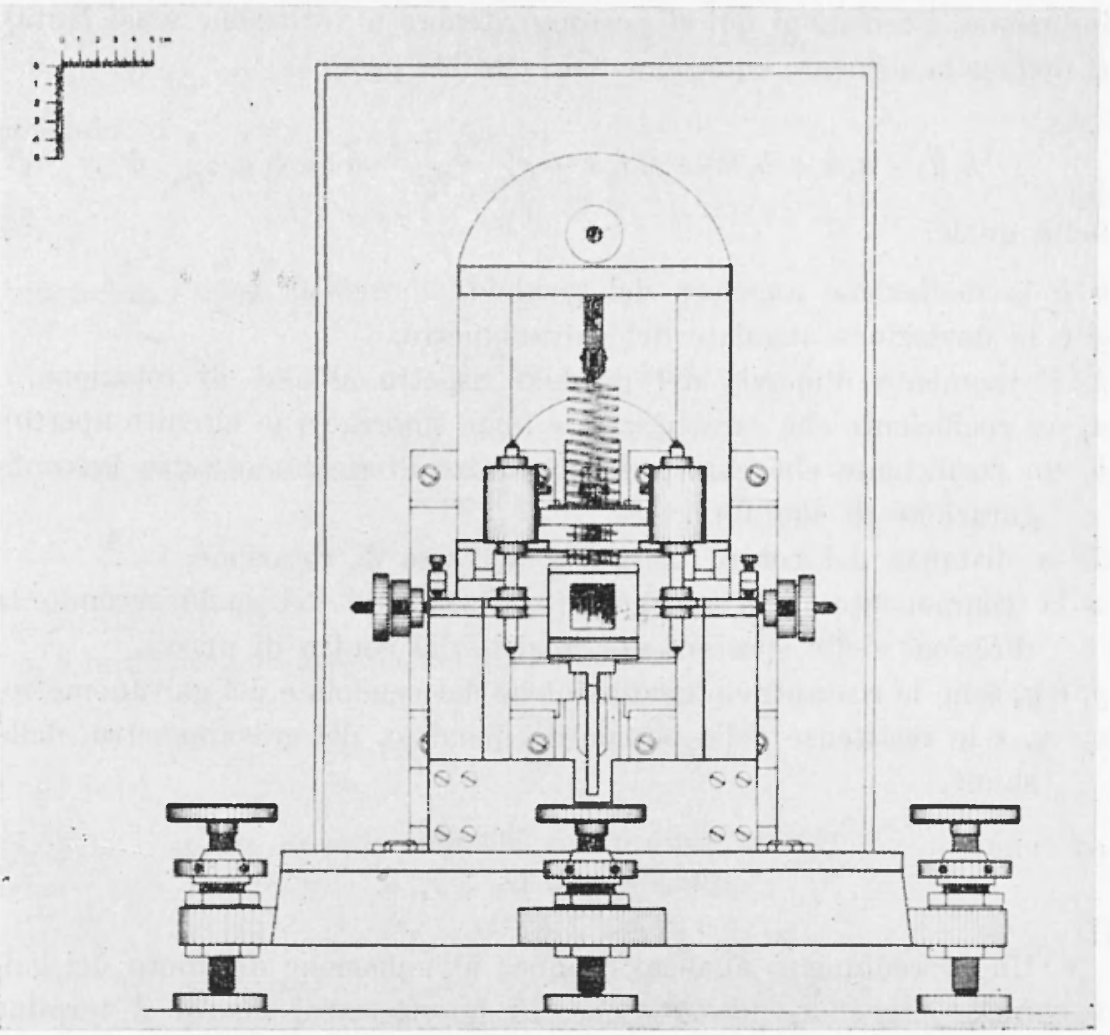

Fig. 6 - Pendolo per la componente verticale visto di dietro.

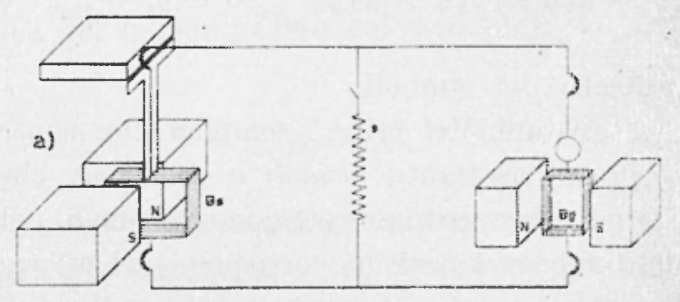

Fig. 7

Lo schema di accoppiamento pendolo-galvanometro è indicato nella fig. 7. Utilizzano l'equazione simbolica della dinamica dei sistemi 
ad un grado di libertà a vincoli privi d'attrito, ritenendo valide le condizioni per le quali sia lecito trascurare i termini non lineari, trascurando le forze elettromotrici di autoinduzione (in quanto, in relazione all'autoinduzione, i fenomeni qui si possono ritenere a variazione assai lenta), si deduce la seguente equazione del moto del pendolo $\left({ }^{5}\right)$ :

$$
k_{1} \ddot{\theta}+a_{1} \theta+b_{1} \theta=M L \ddot{x}-g_{1}^{2} \frac{+s}{R^{2}} \dot{\theta}+g_{1} g_{2} \frac{s}{R^{2}} \dot{\varphi}
$$

nella quale:

$\theta$ è la deviazione angolare del pendolo,

$\varphi$ è la deviazione angolare del galvanometro,

$k_{1}$ il momento d'inerzia del pendolo rispetto all'asse di rotazione, $a_{1}$ un coefficiente che caratterizza le forze smorzanti (a circuito aperto), $b_{1}$ un coefficiente che caratterizza le forze di richiamo verso la configurazione di equilibrio,

$L$ la distanza del centro di massa dall'asse di rotazione,

$x$ la componente dello spostamento traslatorio del suolo secondo la direzione dello spostamento iniziale del centro di massa,

$g_{1}$ e $g_{0}$ sono le costanti elettrodinamiche del pendolo e del galvanometro, $r_{1}, r_{2}, s$ le resistenze delle bobine del pendolo, del galvanometro, dello shunt,

ed infine:

$$
R^{*}=r_{1} r_{2}+\left(r_{1}+r_{2}\right) s
$$

Un procedimento analogo conduce all'equazione del moto del galvanometro, tenendo presente che, in questo caso, manca il termine in $\ddot{x}$, in quanto il centro di massa dell'equipaggio galvanometrico si trova sull'asse di rotazione:

$$
k_{2} \dot{\varphi}+a_{2} \varphi+b_{2} \varphi=g_{1} g_{2} \frac{s}{n^{2}} \dot{\theta}-g_{2}^{2} \frac{r_{1}+s}{D^{2}} \dot{\varphi},
$$

con ovvio significato dei simboli.

Sebbene non esistano dei criteri semplici che consentano di realizzare un sismografo, mediante pendoli a rotazione, che sia rigorosamente insensibile ad una eventuale rotazione del suolo, nel dedurre la [1] si trascurano solitamente i termini corrispondenti all'accelerazione angolare del suolo (termini giro-accelerometrici) e alla velocità angolare (termini girometrici), in considerazione del fatto che, nell'ambito dei movimenti sismici propriamente detti, tali termini sono in genere insignificanti rispetto al termine in $\ddot{x}$ (termine sismometrico). 
Le equazioni [1] e [2] assumono $\left({ }^{4}\right)$ la forma pir̀ espressiva:

$$
\begin{gathered}
\theta+2 \lambda_{1} \omega_{1}\left(\theta-\gamma_{g} \varphi\right)+\omega_{1}^{2} \theta=-\frac{1}{\bar{l}} \ddot{a}, \\
\dot{\varphi}+2 \lambda_{2} \omega_{2}\left(\dot{\varphi}-\gamma_{p} \dot{\theta}\right)+\omega_{2}^{2} \varphi=0,
\end{gathered}
$$

ponendo:

$$
\frac{k_{1}}{M L}=l
$$

(lunghezza ridotta del pendolo);

$$
\frac{b_{1}}{k_{1}}=\omega_{1}
$$

( $\omega_{1}$ è la pulsazione propria del pendolo);

$$
\frac{b_{\Perp}}{k_{2}}=\omega_{2}
$$

( $\omega_{2}$ ̀̀ la pulsazione propria del galvanometro);

$$
\frac{1}{k_{1}}\left(a_{1}+\frac{r_{2}+s}{R^{2}} g_{1}^{2}\right)=2 \lambda_{1} \omega_{1}
$$

( $\lambda_{1}$ è la costante di smorzamento del pendolo accoppiato);

$$
\frac{1}{k_{2}}\left(a_{2}+\frac{r_{i}+s}{n^{2}} g_{0}^{2}\right)=2 \lambda_{2} \omega_{2}
$$

( $\lambda_{2}$ è la costante di smorzamento del galvanometro accoppiato);

$$
g_{1} g_{2} \frac{s}{k_{1} R^{2}}=2 \lambda_{1} \omega_{1} \gamma_{g}
$$

$\left(\gamma_{g}\right.$ è la reazione del galvanometro sul pendolo);

$$
g_{1} g_{2} \frac{s}{l_{2} R^{-}}=2 \lambda_{2} \omega_{2} \gamma_{p}
$$

( $\gamma_{p} \grave{\mathrm{e}}$ la reazione del pendolo sul galvanometro).

La presenza del termine in $\dot{\varphi}$ nell'equazione del moto del pendolo è indizio dell'influenza del moto del galvanometro sul moto del pendolo. Il coefficiente $2 \mu_{\circ}\left(\omega_{2} \gamma_{p}\right.$ di $\theta$, che interviene nell'equazione del moto del galvanometro, non è altro che il coefficiente di trasferimento di Galitzin. 
Tra il moto del suolo, rappresentato dalla funzione $x$, e la risposta del sistema, rappresentata dalla funzione $\varphi$, esiste l'intermediaria funzione 0 , rappresentante la deviazione angolare del pendolo. Per ottenere la relazione diretta tra il movimento del suolo e la risposta del sistema occorre eliminare 0 nelle equazioni $\left[1^{\prime}\right]$ e $\left[2^{\prime}\right]$. Il risultato di tale eliminazione si traduce nella seguente equazione differenziale lineare del $4^{\circ}$ ordine:

$$
\begin{gathered}
\left(\frac{d^{2}}{d t^{2}}+2 \lambda_{1} \omega_{1} \frac{d}{d t}+\omega_{1}^{0}\right)\left(\frac{d^{2}}{d t^{2}}+2 \lambda_{2} \omega_{2} \frac{d}{d t}+\omega_{2}^{2}\right) \varphi- \\
-4 \lambda_{1} \lambda_{2} \omega_{1} \omega_{2} \sigma^{2} \frac{d^{2} \varphi}{d t^{2}}=-\frac{\chi}{l} \frac{d^{3} x}{d t^{3}}
\end{gathered}
$$

nellar quale

$$
\sigma=+\sqrt{\gamma_{g} \gamma_{p}}
$$

è il coefficiente di accoppiamento $\mathrm{e}$

$$
\chi=2 \lambda_{2}()_{2} \gamma_{p}
$$

è il coefficiente di trasferimento. Si noti che

$$
o<\sigma<1
$$

il limite zero si avrebbe soltanto per $k_{1}=\infty$, il che richiederebbe l'uso di masse molto grandi e dimensioni pendolari proibitive; il limite 1 si avrebbe nel caso in cui

$$
a_{1}=a_{2}=0, s=\infty
$$

cioè se lo smorzamento del pendolo e del galvanometro fosse prodotto esclusivamente dal circuito stesso e se, inoltre, lo shunt fosse assente.

Si ottiene una notevole semplificazione introducendo la variabile

essendo

$$
\tau=\omega t
$$

$$
\omega=+\sqrt{\omega_{1} \omega_{2}}
$$

La [10] si trasformerà nell'equazione:

$$
\left(\frac{d^{4}}{d \tau^{4}}+u \frac{d^{3}}{d \tau^{3}}+\dot{v} \frac{d^{2}}{d \tau^{2}}+v \frac{d}{d \tau}+i\right) \dot{\psi}--\frac{\chi}{w l} \frac{d \tau}{d^{3} x},
$$

nella quale

$$
a=2\left(\lambda_{1} v+\lambda_{2} \frac{1}{v}\right)
$$




$$
\begin{gathered}
b=\nu^{2}+\frac{1}{\nu}+4\left(1-\sigma^{2}\right) \lambda_{1} \lambda_{2}, \\
c=2\left(\lambda_{1} \frac{1}{v}+\lambda_{2} v\right),
\end{gathered}
$$

essendo

$$
v=+\sqrt{\frac{\omega_{1}}{\omega_{2}}}=\frac{\omega_{1}}{\omega}=\frac{\omega}{\omega_{2}} .
$$

Le oscillazioni proprie del sistema saranno espresse da

$$
p=\sum_{1}^{+} A_{i} e^{\alpha_{i} \tau}
$$

nella quale le $a_{1}$ sono le radici dell'equazione caratteristica:

$$
a^{4}+a \alpha^{3}+b \alpha^{2}+c \alpha+1=0 .
$$

Tali oscillazioni devono essere necessariamente smorzate e pertanto le $\alpha_{i}$ devono essere negative (se reali) o avere la parte reale negativa (se complesse). Affinché si verifichi questa circostanza, tenendo presente che $a, b, c$ sono costanti positive, è necessario e sufficiente che sia soddisfatta la condizione $\left({ }^{8}\right)$ :

$$
a b c-a^{2}-c^{2}>0 \text {. }
$$

Pertanto, avendo a disposizione un pendolo e un galvanometro aventi assegnate caratteristiche, perché sia possibile effettuare un accoppiamento per realizzare un sismografo elettromagnetico, è necessario che le caratteristiche degli elementi a disposizione siano tali da verificare, attraverso le [11], la condizione [14].

Volendo realizzare un sismografo soddisfacente alle classiche condizioni di Galitzin

$$
\lambda_{1}=\lambda_{2}=1 ; \omega_{1}=()_{2} ; \quad \sigma=0
$$

è necessario che pendolo e galvanometro, oltre ad avere lo stesso periodo, siano portati entrambi all'aperiodicità critica; è necessario, inoltre, che la massa pendolare sia talmente grande da poter trascurare la reazione $\gamma_{g}$ del galvanometro. Tali condizioni, suggerite più che altro per la notevole semplificazione formale che esse determinano, sono assai restrittive ed incomode, specialmente l'ultima. In elïetti si può realizzare la stessa semplificazione formale con condizioni meno restrittive e senza trascurare la reazione galvanometrica. Infatti, tenendo presente che nelle condizioni di Galitzin si ha

$$
a=c=4, \quad b=6,
$$


basta scegliere le caratteristiche $\lambda_{1}, \lambda_{2}, \sigma$ in modo che si abbia:

$$
\begin{aligned}
& a=2\left(\lambda_{1} v+\lambda_{2} \frac{1}{v}\right)=4, \\
& b=v^{2}+\frac{1}{v^{2}}+4\left(1-\sigma^{2}\right) \lambda_{1} \lambda_{2}=6, \\
& c=2\left(\lambda_{1} \frac{1}{v}+\lambda_{2} v\right)=4,
\end{aligned}
$$

per ricondursi alla stessa equazione differenziale:

$$
\left(\frac{d^{4}}{d \tau^{4}}+4 \frac{d^{3}}{d \tau^{3}}+6 \frac{d^{2}}{a}+4 \frac{d}{a} \tau^{2}+1\right) \varphi=-\frac{1}{\omega l} \frac{d^{3} x}{d \tau^{3}} .
$$

Le condizioni [15] si traducono nelle seguenti:

$$
\begin{gathered}
\lambda_{1}=\lambda_{2} \quad ; \quad \frac{2}{\lambda_{1}}=\frac{1}{\nu}+v, \\
\frac{1}{\sqrt{2}}<\lambda_{1}<1, \\
3-2 \sqrt{3}<\nu^{2}<3+2 \sqrt{3}, \\
\frac{1}{\lambda_{1}^{2}}-1=\sigma .
\end{gathered}
$$

Per i sismografi oggetto di questa nota si ha:

$$
v^{2}=2 \quad ; \quad \lambda_{1}=\lambda_{2}=\frac{2}{3} \sqrt{2}
$$

e pertanto è necessario realizzare l'accoppiamento in modo che sia:

$$
\sigma=-\frac{1}{\lambda_{1}^{2}}-1=\frac{1}{8}=0,125
$$

Riferirò in una prossima nota sugli accorgimenti pratici da seguire per far si che $\lambda_{1}, \lambda_{2}, \sigma$ abbiano $i$ valori assegnati perché il sismografo operi nelle condizioni richieste. In quanto segue mi limiterò ad esporre i criteri da seguire per controllare se il sistema è nelle giuste condizioni di regolazione e ad indicare le operazioni necessarie per la determinazione del coefficiente di trasferimento, che, com'è noto, è indispensabile per la costruzione della curva di amplificazione.

Si consideri un movimento brusco del suolo definito come segue:

$$
\left.\begin{array}{l}
x=0 \quad \text { per } \tau<0, \\
\frac{d x}{d \tau}=x_{0} \quad\left(x=x_{\circ} \tau\right) \text { per } \tau \leqslant 0 .
\end{array}\right\}
$$


Dalla [17], che fornisce la risposta del galvanometro, si deducono le seguenti relazioni valevoli per $\tau \leqslant 0$ :

$$
\begin{aligned}
& \frac{d^{4} \varphi}{d \tau^{4}}+4 \frac{d^{3} \varphi}{d \tau^{3}}+6 \frac{d^{2} \varphi}{d \tau^{2}}+4 \frac{d \varphi}{d \tau}+\varphi=-\frac{\chi}{\omega l} \frac{d x^{3}}{d \tau^{3}}=0, \\
& \frac{d^{3} \varphi}{d \tau^{3}}+4 \frac{d^{2} \varphi}{d \tau^{2}}+6 \frac{d \varphi}{d \tau}+4 \varphi+\int_{0}^{\tau} m d \tau=-\frac{\chi}{\omega l} \frac{d^{2} x}{d \tau^{2}}=0, \quad[19] \\
& \frac{d^{2} \varphi}{d \tau^{2}}+4 \frac{d \varphi}{d \tau}+6 \varphi+4 \int_{0}^{\tau} \varphi d \tau+\int_{0}^{\tau} d \tau \int_{0}^{\tau} \varphi d \tau=-\frac{\chi}{\omega l} \frac{d x}{d \tau}=-\frac{\chi}{\omega l} \chi_{0}^{\prime},[21] \\
& \frac{d \varphi}{d \tau}+4 \varphi+6 \int_{0}^{\tau} \varphi d \tau+4 \int_{0}^{\tau} d \tau \int_{0}^{\tau} \varphi d \tau+\int_{0}^{\tau} d \tau \int_{0}^{\tau} d \tau \int_{0} \varphi d \tau=-\frac{\gamma}{\omega \tau} \chi_{0}^{\prime} \tau,
\end{aligned}
$$

dalle quali, supponendo il galvanometro inizialmente in quiete, si traggono le seguenti condizioni iniziali:

$$
\begin{aligned}
& \varphi=0, \\
& \frac{d \varphi}{d \tau}=0, \\
& \frac{d^{2} \varphi}{d \tau^{2}}=-\frac{\ddot{i}}{\omega l} x_{0}^{\prime}, \quad \text { per } \tau=0 . \\
& \frac{d^{3} \varphi}{d \tau^{3}}=4 \frac{\chi}{\omega l} x_{0}^{\prime},
\end{aligned}
$$

Poiché l'equazione caratteristica della [19] ha per radice -1 con molteplicità 4, l'integrale generale è dato da

$$
\varphi=\left(A_{1}+A_{2} \tau+A_{3} \tau^{2}+A_{4} \tau^{3}\right) e^{-\tau} .
$$

Per le [23] si dovrà avere:

$$
\begin{aligned}
& A_{1}=A_{2}=0 \\
& A_{3}=-\frac{1}{2} \quad \underset{\omega l}{\chi} x_{0}^{\prime}, \\
& A_{!}=\frac{1}{6} \frac{\chi}{\omega l} x_{\jmath}^{\prime},
\end{aligned}
$$

e pertanto la risposta del galvanometro sarà espressa da:

$$
\varphi=-\frac{\chi}{\omega_{l} l}-x_{0}^{\prime}\left(\tau^{2}-\frac{1}{3} \tau^{3}\right) e^{-\tau} .
$$


I due valori estremi $\varphi_{1}$ e $\varphi_{2}$ di $\varphi$ si hanno in corrispondenza ai valori

e quindi:

$$
\tau_{1}=3-\sqrt{3} \quad, \quad \tau_{2}=3+\sqrt{3},
$$

$$
\begin{aligned}
& \varphi_{1}=-\frac{\chi \underline{x}_{0}^{\prime}}{(1) l}(2 \sqrt{3}-3) e^{-3} e^{\sqrt{3}}, \\
& \varphi_{2}=\frac{\chi x_{0}^{\prime}}{\text { (1) } l}(2 \sqrt{3}+3) e^{-3} e^{\sqrt{3}},
\end{aligned}
$$

per cui

$$
\frac{\left|\varphi_{1}\right|}{\left|\varphi_{2}\right|}=(7-4 \sqrt{3}) e^{2 \sqrt{3}}=2,29
$$

Quest'ultimo risultato può essere ottenuto col suolo in quiete ed imprimendo al pendolo un impulso istantaneo per mezzo della scarica

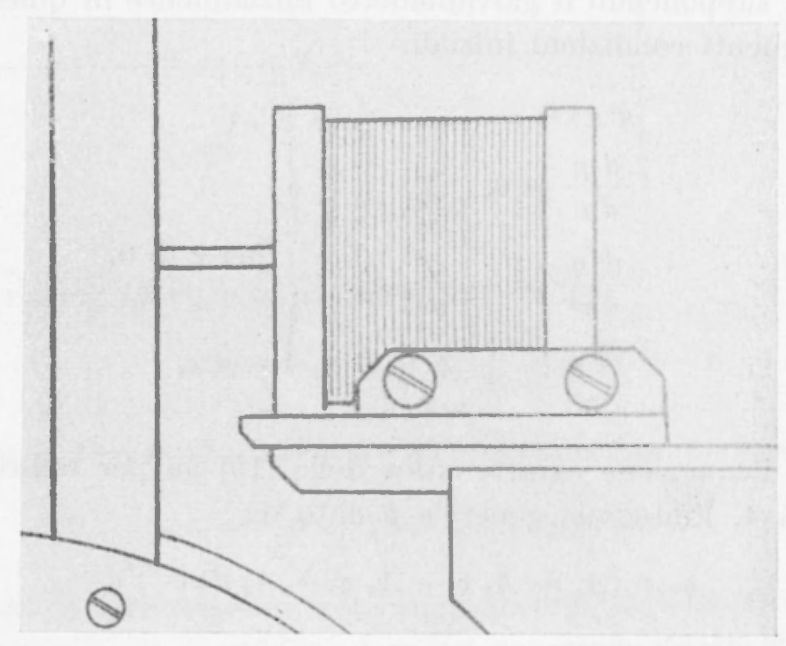

Fig. 8

di un condensatore, avente capacità opportuna, in una bobina situata in modo da abbracciare lungo il proprio asse una appendice di ferro dolce posta lateralmente sul braccio del pendolo (fig. 8). Indicando con $\theta^{\prime}{ }_{o}$ il valore di $d \theta / d \tau$ per $\tau=0$, in tali condizioni si ottiene la seguente soluzione:

$$
\varphi=\hat{\lambda}_{i} \frac{\theta_{n}^{\prime}}{v} \nu_{i}\left(\tau^{2}-\frac{1}{3} \tau\right) e^{-\tau},
$$


cioe:

$$
\varphi=\frac{\chi}{2 \omega} \theta_{0}^{\prime}\left(\tau^{2}-\frac{1}{3} \tau^{3}\right) e^{-\tau} .
$$

I due valori estremi saranno rispettivamente espressi da:

$$
\begin{gathered}
\varphi_{1}=\frac{\chi}{2(1)} \theta_{0}^{\prime}(2 \sqrt{3}-3) e^{-3} e^{\sqrt{3}} \\
\varphi_{2}=-\frac{\chi}{2 \omega} \theta_{0}^{\prime}(2 \sqrt{3}+3) e^{-3} e^{\sqrt{3}}
\end{gathered}
$$

dalle quali segue ancora la [28].

Le [26], [27] o le [31], [32] possono essere utilizzate, attraverso il rapporto [28], soltanto per controllare se il sistema è nelle volute condizioni di regolazione, ma non possono essere adoperate per la determinazione del coefficiente di trasferimento $\chi$. in quanto in tali relazioni

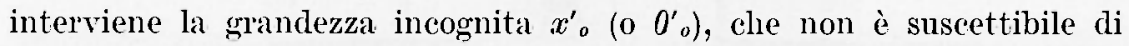
misura diretta. Per tale determinazione si può operare nel modo seguente: si invia nella bobina eccitatrice una corrente di opportuna intensità (col galvanometro disinserito) in modo da produrre una deviazione $0_{0}$ del pendolo, che puó essere misurata per mezzo di un dispositivo scalacannocchiale posto ad una data distanza dallo specchio del pendolo. Si inserisce il galvanometro e, dopo essersi assicurati che lo specchietto del galvanometro si trovi nella sua posizione di zero, si apre il tasto del circuito che alimenta la bobina eccitatrice; il pendolo, abbandonato a se stesso e all'azione della reazione galvanometrica, si muoverà verso la sua posizione di zero con una certa legge che, in questa sede, non interessa stabilire. La risposta de] galvanometro si ottiene ricorrendo ancora allo integrale generale [24], tenendo presente che le condizioni iniziali, in questo caso, sono:

$$
\begin{aligned}
& \varphi=\varphi^{\prime}=\varphi^{\prime \prime}=0, \\
& \varphi^{\prime \prime \prime}=-2 \lambda_{2} v \gamma_{p} \theta_{0}
\end{aligned} \quad \operatorname{per} \tau=0
$$

e pertanto:

$$
\begin{gathered}
A_{1}=A_{2}=A=0 \\
A_{1}=-\frac{1}{3} \lambda_{2} \nu \gamma_{p} a_{11}=-\frac{1}{6} \frac{v}{\omega_{2}} \chi \theta_{0} .
\end{gathered}
$$

Segue:

$$
\varphi=-\frac{1}{6} \frac{v}{\omega_{2}} \chi \theta_{o} \tau^{3} e^{-\tau}
$$


Il massimo di $\varphi$ si avrà per $\tau=3$ e sarà espresso, in valore assoluto da

$$
\left|\varphi_{m}\right|=\frac{9}{2 e^{\nu}} \frac{\nu}{\omega_{2}} \chi 0_{0}
$$

dalla quale si deduce:

$$
\chi=\frac{2 e^{3} \omega_{2}}{9 v} \stackrel{\left|\varphi_{m}\right|}{0_{n}}
$$

Quest'ultima relazione consente la determinazione di $\chi$ attraverso la misura di $\theta_{o}$ (alla scala) e di $\varphi_{m}$ (dalla registrazione fotografica della corrispondente deviazione galvanometrica).

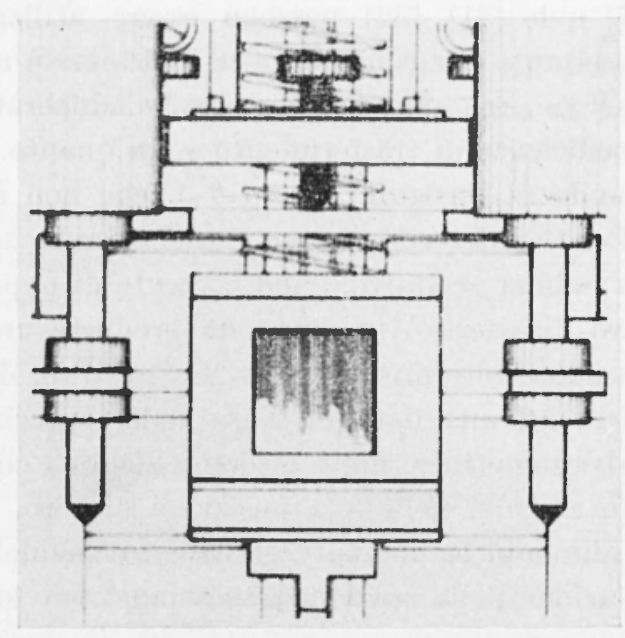

Fig. 9

te necessario osservare che non sempre è possibile partire da valori di $\theta_{0}$ direttamente apprezzabili con la scala, usufruendo di bracci ottici quali normalmente sono consentiti dalle dimensioni del locale che ospita i sismografi. Per sistemi aventi amplificazioni elevate la corrispondente deviazione galvanometrica è troppo ampia per dar luogo ad un tracciato contenibile nel campo utile di registrazione. Per agevolare la lettura sulla scala in un ambiente di modeste dimensioni, lo specchio solidale col braccio pendolare è inclinato (fig. 9), rispetto al piano orizzontale, di $45^{\circ}$; sul supporto del pendolo è montato un altro specchio orizzontale affacciato sul primo; il raggio luminoso proveniente dalla scala subisce una prima riflessione sullo specchio pendolare, una seconda riflessione sullo specchio fisso del supporto che lo rinvia ancora 


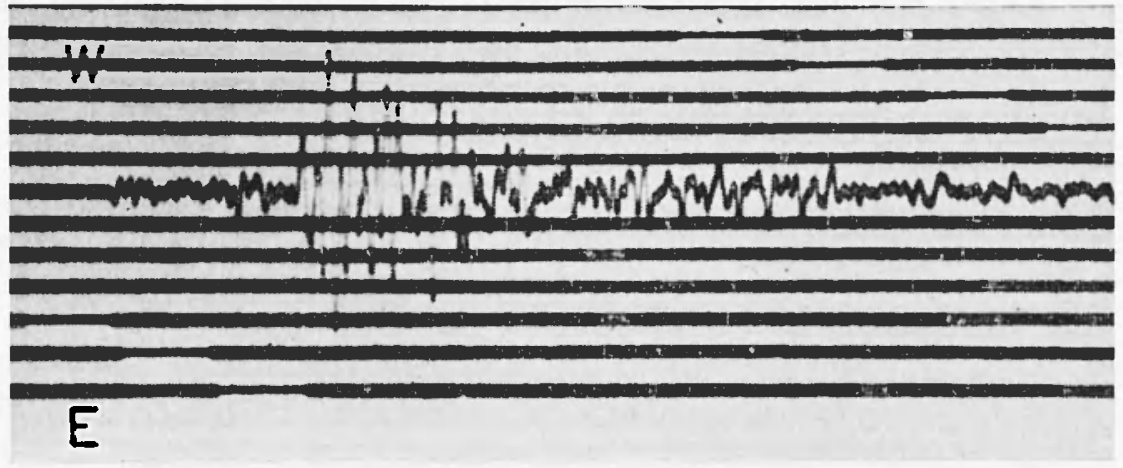

Fig. 10 - Esempio di registrazione di una scossa verificatasi alle falde del Cervino il 15-IX-1958, ottenuta ad Oropa alla distanza di $70 \mathrm{~km}$ ca. Comp. EW.

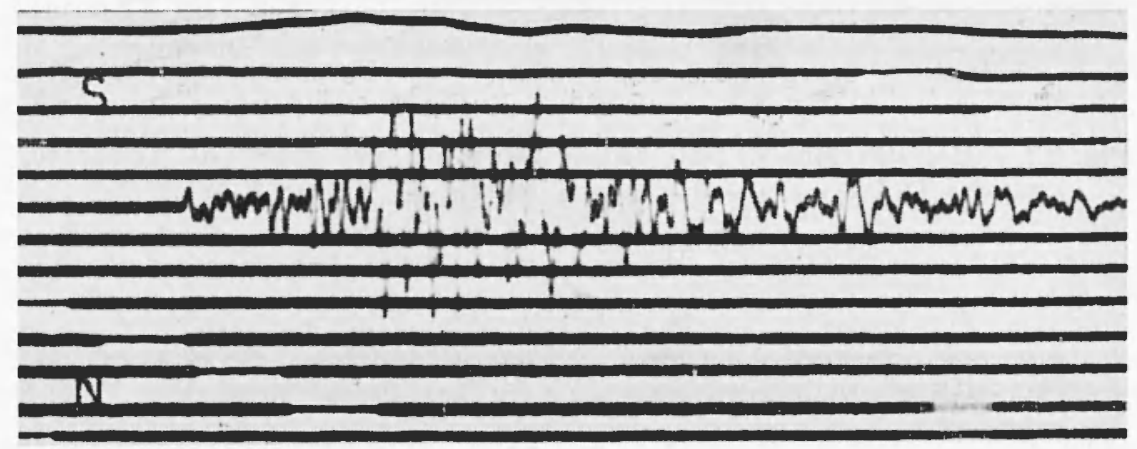

Fig. 11 - Comp. N-S della scossa di cui alla fig. 10.

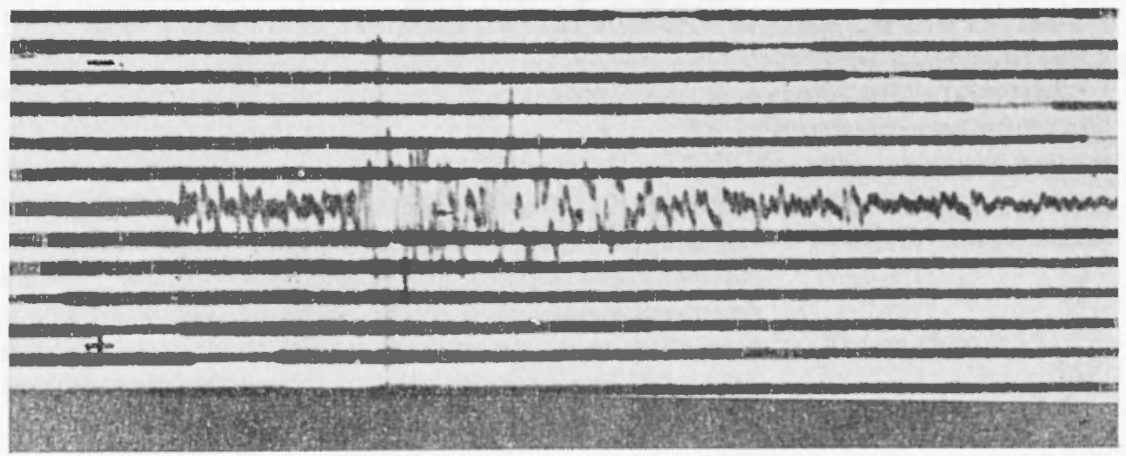

Fig. 12 - Comp. $Z$ della scossa di cui alle fig. 10, 11 


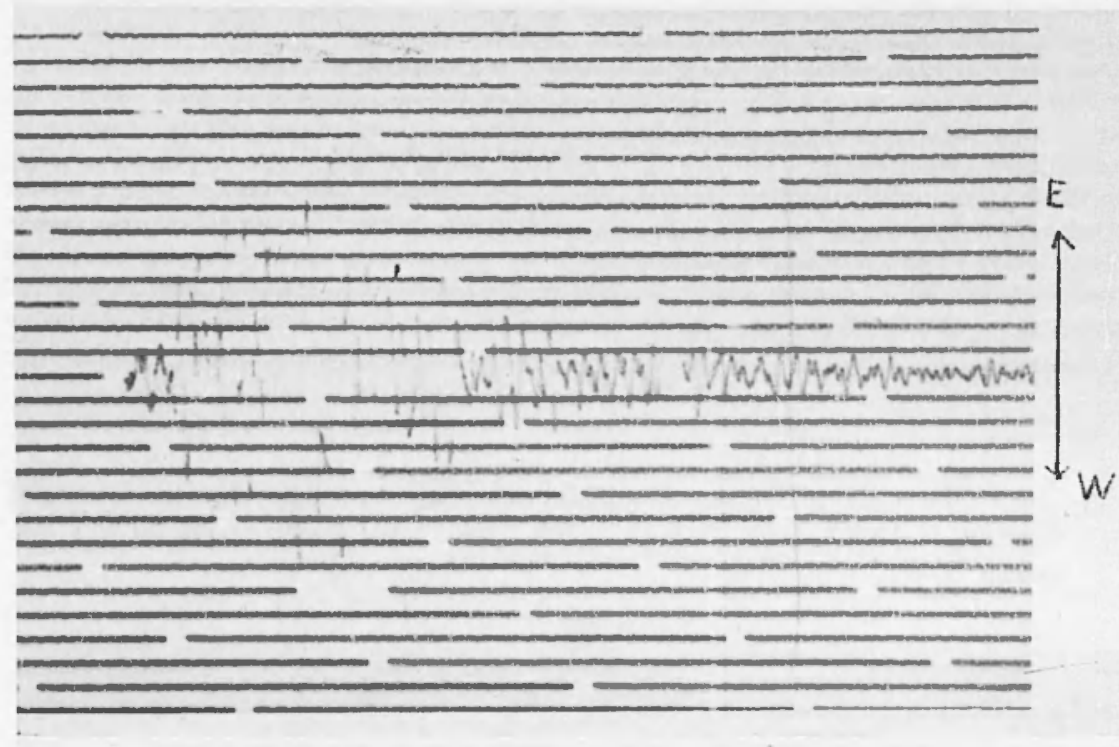

Fig. 13 - Registrazione di una scossa avvenuta nei pressi di Tolmezzo, ottenuta al Vajont alla distanza di $64 \mathrm{~km}$ ca. Comp. E-W.

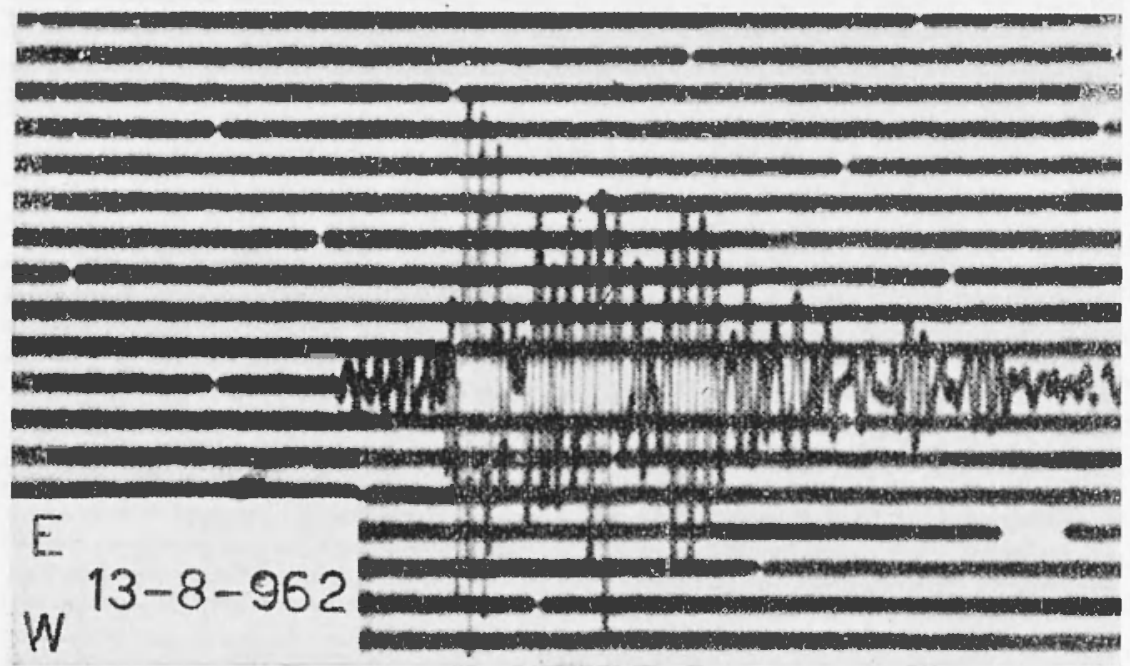

Fig. 14 - Registrazione della stessa scossa ottenuta a Pieve di Cadore, alla distanza di $55 \mathrm{~km}$ ca. Comp. E-TY. 
su quello del pendolo e quindi sul cannoceliale. Con tale dispositivo la formula per la riflessione (per valori di $\theta_{0}$ sufficientenente piceoli)

$$
\theta_{0}=\frac{Z}{2 D}
$$

Sarà sostituita dà

$$
\theta_{0}=\frac{Z}{4 D}
$$

essendo $Z$ la differenza delle letture sulla scalat del camnocelhiale e $D$ la distanzat specchio-scala. Tuttavia, per' ampliticazioni dinamiche snperiori a 7.000 , un tale dispositivo di anplificazione ottica non è sufficiente per apprezzare le deviazioni angolari del pendolo che diano luogo a deviazioni galvanometriche contenibili nel anpo di registazione. In tal caso è necessario determinare $\theta_{0}$ per via indiretta. Prelininamente si costruisce la curva che rappresenta le deviazioni $\theta_{0}$ in funzione dell'intensita di corrente $i$ inviata nella bobina eccitatrice: in tal modo dalla misura di $i$ si puó risalire al valore di $\theta_{0}$.

Istitulo Geofisien e Geodetico della Lniversitic di ILessina. Istituto Nazionale di Geofisica.

\section{BIBLIUGRAFIA}

(1) Gadidzix B., Forlesungen über Seismometrip. Leipzig: Teubner, (1914).

(2) BLnlofF Il., A new vertical seismograph. "Bull. Seismol. Soc. Amer.", 22, (1932).

$\left({ }^{3}\right)$ Wennek F.. I New seismometer Equipped for Electromagnetic Damping and Electromagnetic and Optical Magnification. "Journal of Research ", 2, (1929).

(4) Coulomb J., Grenet G., Nouveaux principes de construction des séismographes électromagnétiques. "Anl1. Phys.", Sér. XL, 3, (1935).

$\left(^{5}\right)$ - Seisnometrie. "Encyclopedia of Physics", XLVII, Geophysics I, springer-Verlag, Berlin, (1956).

$\left.{ }^{(}\right)$Routh H., Advanced rigid dynamics, London, (1905). 